\title{
Development and characterization of rozuvastatin loaded self emulsifying drug delivery system for the effective management of hypolipidemia
}

\author{
Ankit Jain ${ }^{1}$, Naina Dubey ${ }^{1}$, Asmita Gajbhiye ${ }^{2}$, Shailendra Pati ${ }^{3^{*}}$ \\ ${ }^{1}$ Sagar Institute of Pharmaceutical Sciences, Sagar (M.P), India \\ ${ }^{2}$ Department of Pharmaceutical Sciences, Dr H. S. Gour University, Sagar (M.P), India \\ ${ }^{3}$ SVN Institute of Pharmaceutical Sciences, Swami Vivekanand University, Sagar (M.P), India
}

Received: Feb19, 2017, Revised: May 2, 2017, Accepted: Jun 24, 2017

\begin{abstract}
The present work was aimed to develop and characterize rosuvastatin loaded self emulsifying drug delivery system for the effective management of hypolipidemia (RSEDDS) for improving bioavailability, to enhance solubility, to prolong residence time and to provide sufficient amount of drug to a target site in a sustained release manner. Self-emulsifying drug delivery system was prepared by simple emulsification technique, six batches i.e. $\mathrm{fl}$ to $\mathrm{f} 6$ were prepared by varying the concentration of oils, surfactant, co-surfactant and co-solvent and evaluated for the various parameters, e.g. optical microscopy, assessment of self emulsification, emulsification time, droplet size analysis, zeta potential measurement, transmission electron microscopy, viscosity determination, drug content, percentage transmittance, in vitro dissolution study and stability studies. The RSEDDS was optimized and batch F5 was opted for further studies. The drug content of selected batch F5 was found to be $97.65 \pm 1.37 \%$, which suggests that method for encapsulation was effective. The results of in vitro drug release studies showed about $83 \%$ of the drug release within 180 minutes, which exhibit sustained release of drug. There were no significant changes observed in the physical appearance, drug content and in vitro release during stability studies. The studies reveal that the RSEDDS is a potential candidate for sustained release drug delivery which can successfully increase bioavailability.

increasing efficacy.
\end{abstract}

Keywords: Self-emulsifying drug delivery system, rosuvastatin, sustained release, tem, in vitro release

Pharm Biomed Res 2017; 3(2): 1-7

\section{Introduction}

Conventional drug delivery system only a fraction of dose reaches to systemic blood circulation and hence most of the dose is wasted and cause undesirable side effects and toxicity. Also the conventional dosage forms are affected by the gastric environment, $\mathrm{pH}$ conditions, and reaction with the gut walls, GI motility and presence of food in the GI tract. Due to this the drug release pattern from dosage form is affected which in turn affect the therapeutic pattern. This results in longer period of dosing, patient inconvenience and other systemic effects (1). The oral route is the most popular route among all the route of administration. Approximately $40 \%$ of new drug candidates have poor water solubility and the oral delivery of such drugs is frequently associated with low bioavailability, high intra- and inter- subject variability, and a lack of dose proportionality (2). Recently much attention has been paid to lipid based formulations with particular emphasis on selfemulsifying drug delivery system (SEDDS), to improve the oral bioavailability of lipophillic drugs (3).

About $40 \%$ of the drug candidates identified via combinatorial screening programs are poorly water soluble.
The aqueous solubility for poorly water soluble drugs is usually less than $100 \mu \mathrm{g} / \mathrm{ml}$ (4). Especially poorly soluble, highly permeable active pharmaceutical ingredients (BCS Class II drugs) represent the technological challenge, as poor bioavailability is solely caused by poor water solubility resulting in low drug absorption (5). Different techniques have been reported in the literature to achieve better drug dissolution rates. For example (a) reduce the particle size via micronization or nanonization to increase the surface area (b) co-grinding (c) formulation of inclusion complexes (d) solubilisation by surfactants (e) solid dispersions (f) inclusion of the drug solution or liquid drug into soft gelatin capsules such as self-emulsifying drug delivery systems (6). Self-emulsifying drug delivery systems (SEDDs) have gained exposure for their ability to increase solubility and bioavailability of poorly soluble drugs (7). Self-emulsifying drug delivery systems (SEDDs) are mixtures of oils and surfactants, ideally isotropic, and sometimes containing cosolvents, which emulsify spontaneously to produce fine oil-in water emulsions when introduced into aqueous phase under gentle agitation. 
Recently, SEDDS have been formulated using medium chain tri-glyceride oils and non-ionic surfactants, the later being less toxic. $(8,9)$. Upon peroral administration, selfemulsifying formulations distribute readily in the GI tract, and the digestive motility of the stomach and the intestines provides sufficient agitation enough for the spontaneous formation of emulsions. In the case of sparingly soluble drugs that exhibit dissolution rate limited absorption, the SEDDs offers a way to improve the rate and extent of oral absorption and to produce more reproducible blood time profiles (10). Rosuvastatin is a cholesterol-lowering agent widely used to treat hypercholesterolemia. Rosuvastatin is a crystalline powder with a melting point of $151-156{ }^{\circ} \mathrm{C}$, is sparingly soluble in water and poorly absorbed from the gastro-intestinal tract. After oral administration alone, Rosuvastatin is rapidly absorbed maximum plasma concentrations in less than 3 hours, extent of absorption increases in proportion to Rosuvastatin dose. The absolute bioavailability of Rosuvastatin (parent drug) is approximately $20 \%$ and the systemic availability of HMGCoA reductase inhibitory activity is approximately $30 \%$.

Oral bioavailability is the biggest problem of Rosuvastatin calcium as it is slightly water soluble.

Based on these considerations, the objectives of the present work was to develop rosuvastatin loaded self emulsifying drug delivery system for the effective management of hypolipidemia (RSEDDS) to enhance the solubility and improving bioavailability of SEDDS of Rosuvastatin and to administer them through oral route resulting in increasing their clinical efficacy.

\section{Preparation of Self emulsifying drug delivery system}

This involved mixing of different oils, surfactant, co- First weighed amount of rosuvastatin was dissolved in ethanol (AR Grade) by continuous stirring in a beaker until surfactant and co-solvent as shown in table (1).

it totally dissolved. Then oleic acid (AR Grade) was added slowly with continuous stirring into drug-ethanol mixture. In another beaker appropriate amount of PEG-400 (AR Grade) was added to Tween-80 (AR Grade) and mixed properly by continuous stirring with a glass rod. After continuous stirring the mixture of Tween-80 and PEG-400 were added to the drug-ethanol mixture by magnetic stirring at $100 \mathrm{rpm}$ for 30 minute. The formulation of SEDDS was stored in well closed container for its further characterization (11). Selection criteria for preparation of (F5) formulation: The selection of formulation F5 was done on the basis of self emulsification assessment, when compared to other formulations.

\section{Characterization of formulation (F5)}

The opted formulation was selected and characterized for various parameters like optical microscopy, Assessment of self emulsification, Emulsification time, Droplet size analysis, Zeta Potential Measurement, Transmission Electron Microscopy, Viscosity Determination, Drug content, Percentage transmittance, in vitro dissolution study and stability study.

1. Optical microscopy

The opted formulation (F5) of SEDDS observed under optical microscope (Labmed) (Fig 1).

\section{Materials and methods \\ Materials}

Rosuvastatin (AR Grade) was kindly provided as a gift sample by Medley Lab, Jammu, India. Other chemicals used were of AR grade and of $\mathrm{M} / \mathrm{s} \mathrm{CDH}$ Limited, New Delhi, India.

Grade A: Rapidly forming emulsion having a clear or bluish appearance.

Grade B: Rapidly forming, slightly less clear emulsion, having a bluish white appearance.

Grade C: Fine milky emulsion that formed within 2 minutes.

Grade D: Dull, grayish white emulsion having slightly oily appearance that is slow to emulsify longer than 2 minutes.

Grade E: Formulation, exhibiting either poor or minimal emulsification with large oil globules present on the surface.

Table 1 Formulation of SEDDS

\begin{tabular}{lcccccc}
\hline Formulations Drug (rosuvastatin) in $\mathbf{~ m g}$ & $\begin{array}{c}\text { Tween-80 } \\
\text { in } \mathbf{~} \mathbf{L}\end{array}$ & $\begin{array}{c}\text { PEG-400 } \\
\text { in } \mathbf{~ m L}\end{array}$ & $\begin{array}{c}\text { Ethanol } \\
\text { in } \mathbf{~} \mathbf{L}\end{array}$ & $\begin{array}{c}\text { Oleic acid } \\
\text { in } \mathbf{~ m L}\end{array}$ & $\begin{array}{l}\text { Glycerin } \\
\text { in } \mathbf{~ m L}\end{array}$ \\
\hline F1 & 50 & 3.7 & - & 3.6 & 3.7 & 4 \\
\hline F2 & 30 & 3.7 & 4.0 & 3.6 & 3.7 & - \\
\hline F3 & 50 & 3 & - & 4.5 & 3.6 & 4.5 \\
\hline F4 & 30 & 3 & 4.5 & 4.5 & 3.6 & - \\
\hline F5 & 50 & 5 & 2.5 & 2.5 & 5 & - \\
\hline F6 & 30 & 5 & - & 2.5 & 5 & 2.5 \\
\hline
\end{tabular}




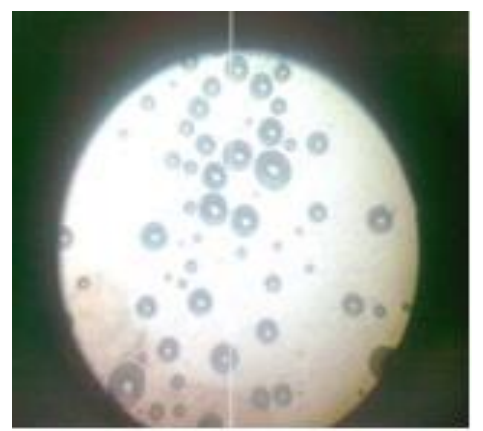

Figure 1 Photograph of formulation (F5) of SEDDS of rosuvastatin under optical microscope

Table 2 Assessment of self emulsification for various SEDDS formulations

\begin{tabular}{ll}
\hline Formulation & Grade \\
\hline F1 & C \\
\hline F2 & B \\
\hline F3 & D \\
\hline F4 & C \\
\hline F5 & A \\
\hline
\end{tabular}

Table 3 Assessment of self emulsification, emulsification time, droplet size and zeta potential

\begin{tabular}{lllll}
\hline Formulation & $\begin{array}{l}\text { Self } \\
\text { Emulsification }\end{array}$ & $\begin{array}{l}\text { Emulsification } \\
\text { Time }\end{array}$ & $\begin{array}{l}\text { Droplet Zetapotential } \\
\text { Size }\end{array}$ & \\
\hline F5 & Grade A & $19 \pm 5.51 \mathrm{sec}$ & $125.89 \mathrm{~nm}$ & $-25.7 \mathrm{mV}$ \\
\hline
\end{tabular}

\section{Emulsification time}

The emulsification time of SEDDS was determined according to USP XXIII, dissolution apparatus II. $0.5 \mathrm{~g}$ of the SEDDS formulation (F5) was introduced into $250 \mathrm{ml}$ of $0.1 \mathrm{~N} \mathrm{HCl}$ in $500 \mathrm{ml}$ conical flask under action of magnetic stirrer (Jaico) rotating at constant speed $(50 \mathrm{rpm})$ and emulsification time was noted (Table 3) $(13,14)$.

3. Droplet size analysis

Droplet size determines the rate and extent of drug release as well as the stability of the emulsion. Formation of SEDDS, which are stable, isotropic and clear o/w dispersions, takes place on reduction of the globule size. SEDDS formulation (F5) was diluted to $100 \mathrm{~mL}$ with distilled water in a flask and is mixed gently by inverting the flask. The droplet size was determined by dynamic light scattering (DLS) technique using Zetasizer (Zetasizer Ver. 6.01, Malvern Instruments, (UK) (Table 3) (15).

\section{Zeta potential measurement}

The emulsion stability is directly related to the magnitude of the surface charge. The magnitude of the zeta potential gives an indication of the potential stability of the colloidal system. If all the particles have a large negative or positive zeta potential they will repel each other and there is dispersion stability. The zeta potential of the diluted SEDDS formulation was measured using a (Malvern Zetasizer $3000 \mathrm{HS}$ ). The SEDDS were diluted with a ratio of 1:20 v/v with distilled water and mixed for 1 min using a magnetic stirrer and recorded the result (Table 3) (Figure 2) $(16,17,18)$.

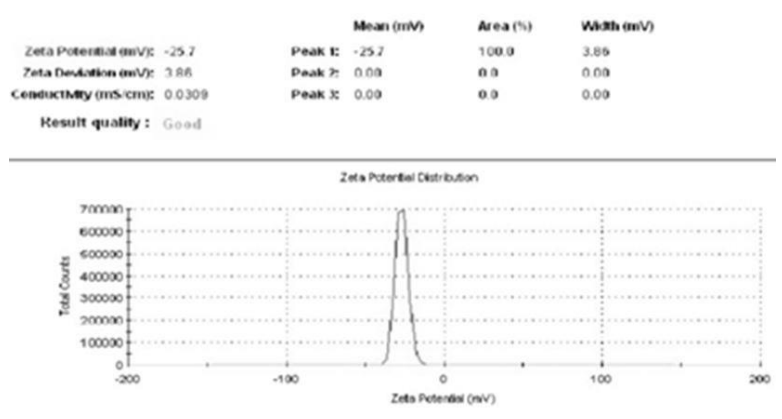

Figure 2 Zeta potential of SEDDS formulation (F5).

\section{Transmission electron microscopy}

SEDDS formulation F5 was diluted with distilled water 1:30 and mixed by gentle shaking using a (Hitachi H-7500, $120 \mathrm{kV}$ ). Copper grids are allowed to stand on for 60 seconds on which one drop of sample obtained after dilution was deposited. Filter paper is used to remove excess fluid and then the grid was stained in $1 \%$ phosphotungstic acid solution for 30 seconds. (Figure 3) (19).

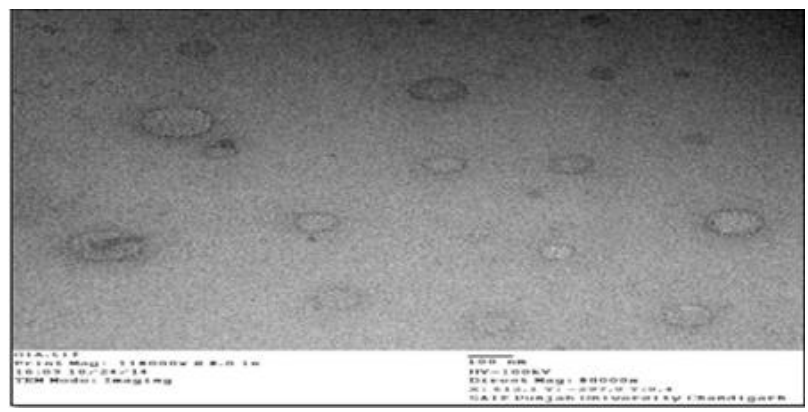

Figure 3 TEM photomicrograph of SEDDS of rosuvastatin $(\mathrm{F} 5)$.

6. Viscosity determination

Viscosity study is necessary for SEDDS to characterize the system physically and to control its stability. The viscosity of the Rosuvastatin SEDDS is crucial in determining its ability to be filled in hard or soft gelatin capsules. If the system has very low viscosity, it may enhance the 
probability of leakage from the capsule and the system with very high viscosity may create problem in pour ability.

SEDDS formulation (F5) of rosuvastatin (1 mL) was diluted with the distilled water in a beaker with constant stirring on magnetic stirrer. Viscosity of the resultant emulsion and initial SEDDS was measured using Brookfield viscometer (DV-III ultra Brookfield) using spindle RV-6 at $100 \mathrm{rpm}$ at $25 \pm 0.5{ }^{\circ} \mathrm{C}$. The data of viscosity of SEDDS formulation F5 was recorded (Table 4) $(20,21)$.

Table 4 Assessment of Viscosity, Drug content and \% transmittance

\begin{tabular}{cccc}
\hline Formulation & Viscosity & Drug content & \%transmittance \\
\hline F5 & $12.2 \pm 0.2 \mathrm{cP}$ & $97.65 \pm 1.37$ & $97.45 \pm 1.78$ \\
\hline
\end{tabular}

\section{Drug content}

The drug content of rosuvastatin SEDDS formulation (F5) was measured using UV spectroscopic method. The drug content uniformity was determined by preparing 10 $\mu \mathrm{g} / \mathrm{ml}$ of aliquot of SEDDS sample using methanol as solvent. The samples were suitably diluted and the absorbance of the solutions was measured at $240 \mathrm{~nm}$ using UV-Visible spectrophotometer (EI double beam spectrophotometer 1372 UV-Spectrophotometer) against methanol as a blank. The amount of Rosuvastatin was estimated by using standard calibration curve of the drug. The data of percent drug content in SEDDS formulation (F5) was recorded in the table (Table 4) (22).

8. Percentage transmittance

Percent transmittance proved the transparency of formulation. The percent transmittance of the system is measured at particular wavelength using UV spectrophotometer (EI double beam UV-VIS spectrophotometer UV/Visible model 1372) by using distilled water as blank (20).

A total of $1 \mathrm{~mL}$ SEDDS formulation was diluted 100 times with distilled water. Percentage of transmittance was measured spectrophotometrically (EI double beam spectrophotometer 1372 UV Spectrophotometer) at $560 \mathrm{~nm}$ using water as a blank (Table 4).

9. Invitro dissolution study

The quantitative in-vitro drug release from formulation was studied to assess if self emulsifying properties remain consistent. The USP XXII, dissolution apparatus (Electrolab TDT-061) used to study the release of the drug from the oil in the aqueous system. Hard gelatin capsule containing SEDDS was tied to paddle to prevent the capsule from floating, $900 \mathrm{ml}$ dissolution media (standard phosphate buffer solution $\mathrm{pH}$ 7.4) were used (18).

To compare different SEDDS, dissolution studies were done at $37 \pm 0.5^{\circ} \mathrm{C}$, using paddle rotating at $75 \mathrm{rpm}, 1 \mathrm{ml}$ sample was withdrawn at 30, 60, 90, 120, 150, 180 minutes. The sample volume of fresh media replaces the withdrawn sample. Sample was filter through whatmann filter paper and analyzed spectrophotometrically (EI double beam UVVIS spectrophotometer UV/Visible model 1372) at 240 $\mathrm{nm}$. The drug release from the SEDDS formulation was found to be significantly higher as compared with that of pure drug and marketed preparation (Crestor® $10 \mathrm{mg}$ ) (Figure 4).

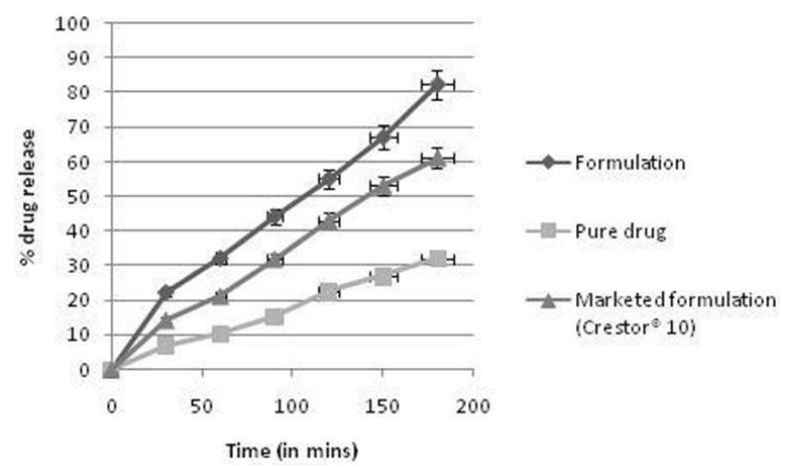

Figure 4 Percentage drug release of F5, pure drug and marketed formulation

10. Stability studies

The optimized formulation (F5) selected for stability testing under storage condition at $4 \pm 1{ }^{\circ} \mathrm{C}, 25 \pm 2{ }^{\circ} \mathrm{C}$ (room temperature) and $40 \pm 1{ }^{\circ} \mathrm{C}$ (Thermostatic oven). Formulation (F5) was stored in screw capped, amber colored small glass bottles at $4 \pm 1{ }^{\circ} \mathrm{C}$, room temperature $\left(25 \pm 2{ }^{\circ} \mathrm{C}\right)$ and $40 \pm 1{ }^{\circ} \mathrm{C}$. Analysis of the sample was made for $\%$ drug content after a period of 15, 30, 45 and 60 days. Subsequent change in $\%$ Drug content of the formulations stored at $4 \pm 1{ }^{\circ} \mathrm{C}$, at room temperature $25 \pm$ $2{ }^{\circ} \mathrm{C}$ and at $40 \pm 1{ }^{\circ} \mathrm{C}$ (Thermostatic oven) was determined after a definite period of time of $15,30,45$, and 60 days (Table 5) (14).

Table 5 Effect of Storage on \% Residual Drug Content at different temperatures

\begin{tabular}{ccccc}
\hline S.no & $\begin{array}{c}\text { Time in } \\
\text { Days }\end{array}$ & \multicolumn{2}{l}{$\begin{array}{l}\text { \% Residual drug content on } \\
\text { storage at temperature }\end{array}$} \\
\cline { 3 - 5 } & & $4 \pm 1^{\circ} \mathrm{C}$ & $25 \pm 1{ }^{\circ} \mathrm{C}$ & $40 \pm 1^{\circ} \mathrm{C}$ \\
\hline 1. & 0 & $97.6 \pm 1$ & $97.6 \pm 1$ & $97.6 \pm 1$ \\
\hline 2. & 15 & $97.6 \pm 1$ & $97.4 \pm 1$ & $97.4 \pm 1$ \\
\hline 3. & 30 & $97.3 \pm 1$ & $96.8 \pm 1$ & $96.5 \pm 0.5$ \\
\hline 4. & 45 & $96.9 \pm 0.2$ & $96.1 \pm 0.3$ & $95.8 \pm 0.9$ \\
\hline 5. & 60 & $96.5 \pm 0.6$ & $95.7 \pm 0.7$ & $95.2 \pm 0.6$ \\
\hline
\end{tabular}




\section{Results}

The selection of formulation F5 was done on the basis of self emulsification assessment, when compared to other formulations; the F5 formulation formed a rapidly forming emulsion having a clear or bluish appearance that was the formulation of Grade-A category. In the above formulation design the F5 formulation was finalized for the further studies for characterization under various parameters. (Table 2 and 3) The self emulsification assessment of SEDDS showed that the preparation was of Grade A that is a rapidly forming emulsion having a clear or bluish appearance. It was observed that an increase in the proportion of oil in the formulation resulted in decreasing self-emulsification time.

The opted formulation (F5) of SEDDS observed under optical microscope (Labmed) and it was found that the developed formulation contained the droplets in emulsion (Figure 1).

The emulsification time of SEDDS was $19 \pm 5.51$ seconds which resulted in good tendency for emulsification. (Table 3)

The droplet size of SEDDS (F5) formulation was found to be $125.89 \mathrm{~nm}$ which explained that the smaller droplet size presents large surface area for drug absorption. (Table 3) and the zeta potential value of SEDDS (F5) was found to be $-25.7 \mathrm{mV}$ negative charge indicates that the emulsion particles were stable (Table 3) (Figure 2).

The TEM photograph shows the surface morphology of the SEDDS (F5) as seen in (Figure 3) the nanosized droplets as discreet particles can be seen in the TEM analysis is evidence to show that the adsorption onto solid carrier was good as no oil droplets are visible.

Viscosity of SEDDS (F5) was found to be $12.2 \pm 0.2(\mathrm{cP})$. Thus it showed o/w emulsion where water remains as external phase and viscosity of SEDDS is near to water which indicated that Rosuvastatin emulsion on dilution with the fluid its viscosity getting decreased and thereby absorption will be faster (Table 4).

The percentage drug content was found to be $97.65 \pm 1.37$ which is maximum and thus resulted in maximum drug release (Table 4).

The result of percentage transmittance was shown $97.45 \pm$ 1.78 (Table 4). This result indicated the high clarity of SEDDS. The greater the particle size, oil globules may reduce the transparency of micro emulsion and thereby values of percentage transmittance.

The formulation of SEDDS (F5) showed greater extent of drug release that is in 180 mins the drug release was $83 \%$ when compared to pure drug (32\%) and marketed formulation $(61 \%)$. The results suggested the potential use of SEDDS for oral administration of Rosuvastatin (Figure 4).

Accelerated stability studies only serve as tool for formulation screening and stability issues related to shipping or storage at room temperature. The results of the stability samples withdrawn at the end of 15 days, 30 days, 45 days and 60 days are shown in (Table 5 ) at various temperature ranges. All the samples withdrawn at different time intervals formed clear dispersion and none of the formulation showed any drug precipitation and thus the formulation was considered to be stable.

A progressive decrease in the emulsion $\%$ drug content has been observed in the samples withdrawn at different time intervals which may be due to aggregation of globules.

Rosuvastatin loaded SEDDS (F5) was subjected to stability studies. The formulation was stored at $4 \pm 1{ }^{\circ} \mathrm{C}$, at $25 \pm 2$ ${ }^{\circ} \mathrm{C}$ (Room temperature) and at $40 \pm 1{ }^{\circ} \mathrm{C}$ (Thermostatic oven). From the results, it was found that $\%$ drug content had shown that formulation was more stable at $25 \pm 2{ }^{\circ} \mathrm{C}$ rather than $4 \pm 1{ }^{\circ} \mathrm{C}$ and $40 \pm 1{ }^{\circ} \mathrm{C}$ (Thermostatic oven) storage conditions. So it can be said that the formulation is more stable at $25 \pm 2{ }^{\circ} \mathrm{C}$ for further use (Table 5).

\section{Discussion}

SEDDSs are isotropic mixtures of oils and surfactants, sometimes it contains co-solvents and it can be used for the design of formulations in order to improve the oral absorption of highly lipophillic compounds.

Rosuvastatin is a hydrophobic and highly permeable drug which belongs to class II of biopharmaceutical classification system (BCS). Low aqueous solubility of Rosuvastatin leads to high variability in absorption after oral administration. The present study was carried out for the formulation development of Rosuvastatin loaded selfemulsifying drug delivery system (SEDDS) with the aim to enhance its solubility as well as oral bioavailability.

The opted formulation (F5) of SEDDS observed under optical microscope (Labmed) and it was found that the developed formulation contained the droplets in emulsion (Figure 1).

The self emulsification assessment of SEDDS showed that the preparation was of Grade A that is a rapidly forming emulsion having a clear or bluish appearance (Table 2 and $3)$. It was observed that an increase in the proportion of oil in the formulation resulted in decreasing self-emulsification time.

The RSEDDS should disperse completely and quickly when subjected to aqueous dilution under mild agitation. 
The emulsification time study showed that the optimized formulation employed could emulsify within $19 \pm 5.51$ seconds, which suggests rapidity of the formulation. The emulsification time of SEDDS was which resulted in good tendency for emulsification (Table 3). The droplet size of the emulsion is a crucial factor in self-emulsification performance because it determines the rate and extent of drug release as well as absorption. The droplet size of SEDDS (F5) formulation was found to be $125.89 \mathrm{~nm}$ which explained that the smaller droplet size presents large surface area for drug absorption (Table 3). In general, a zeta potential value of $\pm 30 \mathrm{mV}$ is sufficient for stability. The optimized formulation posses a zeta potential of $-25.7 \pm$ 0.42 , which means that it does not comply with requirement of stability. But, it has been found in some of the research articles that a zeta value in between $-20 \mathrm{mV}$ and $-11 \mathrm{mV}$ leads to threshold agglomeration. Hence, the optimized formulation of RSEDDS with a zeta potential $-25.7 \mathrm{mV}$ would not exhibit threshold agglomeration. Such kind of instability will only be observed on long-term storage (Table 3) (Figure 2).

The TEM photograph showed spherical surface morphology of SEDDS (F5) which resulted in higher drug loading (Figure 3).

Viscosity of SEDDS (F5) was found to be $12.2 \pm 0.2 \mathrm{cP}$, thus it showed $\mathrm{o} / \mathrm{w}$ emulsion where water remains as external phase and viscosity of SEDDS is near to water which indicated that Rosuvastatin emulsion on dilution with the fluid its viscosity getting decreased and thereby absorption will be faster (Table 4).

The amount of the drug content in the optimized formulation F5 is $97.65 \pm 1.37$ of the added amount indicating the suitability of the system for high entrapment in the internal phase. The high value of percentage transmittance $97.45 \pm 1.78(96.21 \%)$ indicates that the system is optically clear which a prerequisite for emulsions (Table 4). The greater the particle size, oil globules may reduce the transparency of micro emulsion and thereby values of percentage transmittance.

The formulation of RSEDDS (F5) showed greater extent of drug release that is in 180 mins the drug released was $83 \%$ when compared to pure drug (32\%) and marketed formulation $(61 \%)$. The results suggested the potential use of SEDDS for oral administration of Rosuvastatin (Figure 4). Rosuvastatin loaded SEDDS (F5) was subjected to stability studies. The formulation was stored at $4 \pm 1{ }^{\circ} \mathrm{C}$, at $25 \pm 2{ }^{\circ} \mathrm{C}$ (room temperature) and at $40 \pm 1{ }^{\circ} \mathrm{C}$ (thermostatic oven). The formulation was physically stable as evidenced by the visual observation, and the absence of signs of instability such as phase separation, flocculation or precipitation. From the results, it was found that $\%$ drug content had shown that formulation was more stable at $25 \pm$ $2{ }^{\circ} \mathrm{C}$ rather than $4 \pm 1{ }^{\circ} \mathrm{C}$ and $40 \pm 1{ }^{\circ} \mathrm{C}$ (thermostatic oven) storage conditions (Table 5).

\section{Conclusion}

In conclusion, the proposed system liquid SEDDS showed incredible potential and good self emulsification efficiency, having globule size in nanometer range which is physiologically stable with improved solubility and bioavailability of otherwise poorly soluble BCS class II drug rosuvastatin.

In summary, our findings indicated that the developed formulation was found to show a significant improvement in terms of the drug release with above $83 \%$ release of drug within 180 mins. Thus, achieved the main objective of study to achieve a better dissolution rate which would further help in enhancing oral bioavailability. The SEDDS may be used as a vital tool in reducing the dose size and improving oral bioavailability of poorly water soluble drugs.

\section{Future Prospects}

An optimized Rosuvastatin loaded formulation consisting of oleic acid, tween 80 and PEG 400 offers the advantage of good solubilisation of Rosuvastatin. The major limitation of lipophillic drugs regarding its solubility in GIT could be overcome by delivering these drugs through selfemulsifying systems. Thus, this novel delivery system has made easy the delivery of lipophillic drugs orally which increases its bioavailability due to its small particle droplets. The studies confirmed that SEDDS can be used as a possible alternative to conventional oral formulation of BCS class II drugs (poorly water soluble drugs) and can be explored as a potential drug carrier for dissolution enhancement of Rosuvastatin and other insoluble drugs.

\section{Acknowledgments}

We are grateful to Principal, Sagar Institute of Pharmaceutical Sciences, Sagar, for providing us necessary facilities to stock out the research work. The authors are also grateful to Medley Laboratories, Jammu for providing Rosuvastatin and highly obliged to Scan Laboratories, Bhopal for their contribution in completing the work.

\section{Author's contribution}

All authors have contributed in the studies performed and in the preparation of manuscripts. 


\section{Conflict of interest}

The authors report no conflicts of interest.

\section{Financial disclosure}

No financial supports have been granted by any agency to conduct these studies.

\section{References}

1. Jain NK, Sharma SN. A text book of professional pharmacy. 5th edition published by N. K. Jain for VallabhPrakashan New Delhi 2008;1-11.

2. Sharma S, Sharma AD, Chauhan B, Sanwal R. Self Emulsifying Drug Delivery Systems: A Modern Approach for Delivery of Lipophilic Drug. Ordonear Research Library 2011; 1:121-2.

3. Jing LT, Jin S, Zhong GH. Self-Emulsifying Drug Delivery Systems: Strategy for Improving Oral Delivery of Poorly Soluble Drugs. Curr Drug ther 2007; 2:85-93.

4. Ngiik T, Amal AE. Effects of liquid solid formulations on dissolution of naproxen. Eur J PharmBiopha 2009;73:373-84.

5. Hentzschel CM, Alnaief M, Smirnova I, Sakmann A, Leopold CS. Enhancement of griseofulvin release from liquid solid compacts. Eur J PharmBiopha 2011;73:1-6.

6. Vijay KN, Ramarao T, Jayaveera KN. Liquisolid compacts: A novel approach to enhance bioavailability of poorly soluble drugs. Int JPharm BioSci 2011;1:89-102.

7. Sachan R, Khatri K, Kasture SB. Self-Eumlsifying Drug Delivery System: A Novel Approach for enhancement of bioavailability. IntJ Pharm Tech Res 2010;2:1738-45.

8. Patel NN, Rathva SR, Shah VH, Upadhyay UM. Review on self emulsifying drug delivery system: Novel approach for solubility enhancement. IntJPharm Resallied sci2012;1:1-12.

9. Patel PA, Chaulang GM, Akolkotkar A, Mutha SS, Hardikar SR,Bhosale AV. Self emulsifying drug delivery system: A Review. Research J.Pharm. and Tech. 2008;1:313-23.

10. Jingling T, Jin S, Fude C, Zhonggui H. Preparation of Selfemulsifying Drug Delivery Systems of Ginkgo biloba Extracts and In vitro Dissolution Studies. AsianJ Traditional Medicines 2006;1: 1-4.
11. Khoo SM, Humberstone AJ, Porter CJ, Edwards GA, Charman WN. Formulation design and bioavailability assessment of lipidic self- emulsifying formulations of halofantrine. Int $\mathbf{J}$ Pharm 1998;167:155-64

12. Naisarg DP. Self-emulsifying drug delivery system: A novel approach. Int J CurPharmRes 2012;4:18-23.

13. Balakrishnan P, Lee BJ, Oh DH, Kim JO, Hong MJ, Jee JP Enhanced oral bioavailability of coenzyme Q10 by a novel solid self-emulsifying drug delivery system. Int J Pharm2009; 74:66-72.

14. Bhatt PP. Osmotic Drug Delivery Systems for Poorly Soluble Drugs, The Drug Delivery Companies Report Autumn/Winter 2004, 26-29.

15. Kavita S, Ashu S, Singh SK, Saloni K. Self emulsifying drug delivery system: A tool in solubility enhancement of poorly soluble drugs. Indo Global J Pharm Sci 2012;2:313-32.

16. Dhomne, Formulation and evaluation of solid self emulsifying drug delivery system for lipophyllic drugs, International Journal of Pharmaceutical Sciences Review and Research. 2012; 3:32-36.

17. Himani B, Seema B, Mayank Y, Vinod S, Mamta S. Self emulsifying drug delivery system: An approach to enhance bioavailability. International Journal of Pharma. Research and Development 2008;3:59-75.

18. Pathak CV, Gujarathi NA, Rane BR, Pawar SP.A review on self micro emulsifying drug delivery system. IntJ Pharm Sci 2013; 4:3628-48

19. Liang M, Davies NM, Toth I. Increasing entrapment of peptides from water in oil Microemulsions by copolymerization. Int J Pharm 2008; 362:141-6.

20. Yogeshwar G, Vandana B. SMEDDS of Glyburide: Formulation, in vitro evaluation, and stability studies. AAPS Pharm Sci Tech 2009;10:482-7.

21. Tayal A, Jamil F, Sharma R, Sharma S. Self emulsifying drug delivery system: A review. International Research Journal of Pharm 2012;3:32-6.

22. Sharma S, Bajaj H, Bhardwaj P, Sharma AD, Singh R.Development and Characterization of self emulsifying drug delivery system of poorly water soluble drug using natural oil. Acta Pol Pharm 2012;69:713-7. 